\title{
Prevalence of dermatoses among hairdressers and beauticians in Srinagar, the capital city of Kashmir, India
}

\section{Iffat Hassan, Faizan Younus Shah, Najam-u Saqib, Manzoor Ahmad Bhat, Aaqib Aslam Shah, Yaqzata Bashir, Irfan Tasaduq, Uzair Khursheed Dar, Shuhaab Ahmad Shah}

Department of Dermatology, Venereology \& Leprosy, Government Medical College, Srinagar, India

Corresponding author: Prof. Iffat Hassan, MD, E-mail: hassaniffat@gmail.com

\begin{abstract}
Background: Skin disorders among hairdressers and beauticians have been recognized as a common problem, owing to the prolonged exposure to a variety of irritants and allergens. Objectives: The aim was to study the prevalence of dermatoses among hairdressers and beauticians in Srinagar, the capital city of Kashmir, India. Methods: The study was conducted on 100 respondents from 57 salons and parlors located within the borders of the city. Detailed history taking and complete examination were performed during personal visits. Patch tests were performed with the Indian standard series on all participants. Results: The mean age of the study sample was $24.3 \pm 6.3$ years. $54 \%$ of the studied cases were found to suffer from one or more skin disorders. Hand eczema was the most commonly encountered dermatosis, observed in $32 \%$ of the cases. Three patterns of hand eczema were recognized: classic (14\%), interdigital (6\%), and threading (8\%). Callosities were seen in $19 \%$ of the cases. We employed the term scissor nodule for the typical pattern of a callosity caused by the finger rings of scissors. Nail staining was encountered in 19\%. The most common allergens leading to a positive patch test were found to be paraphenylenediamine (PPD) in thirteen cases, followed by a fragrance mix in nine, nickel sulfate in nine, thiuram in three, and formaldehyde in one. Conclusion: Skin disorders are common among hairdressers and beauticians. Raising awareness of these disorders and methods of their prevention among this group is imperative.
\end{abstract}

Key words: Beauticians; Hairdressers; Dermatoses; Prevalence; Kashmir, India

\section{INTRODUCTION}

The history of hairdressing and beauty salons dates back thousands of years. Ancient Egyptians were well versed in the art of hair dyeing with vegetable dyes as early as $5000 \mathrm{BC}[1]$. Hairdressers were held in great esteem in some cultures of Africa, owing to the belief that a person's spirit occupies their hair. The word hairdresser is used for the first time in Europe during the $17^{\text {th }}$ century. While influential and wealthy families had personal barbers in their homes, community barbershops sprouted for the less privileged. Beauty salons became popular during the $20^{\text {th }}$ century. Focused on women, these places provided services such as facials and hairstyling, additionally serving as social spaces, allowing women to socialize. The role of hairdressers and beauticians in the $21^{\text {st }}$ century has expanded from traditional hairdressing and cleaning to a myriad of cosmetic procedures such as conditioning, bleaching, dyeing, waving, straightening, waxing, cleansing, and providing spa treatments.

Hairdressers and beauticians bear the increased risk of developing occupational dermatoses due to exposure to a variety of irritants and sensitizers, such as shampoos, conditioners, dyes, bleaches, waxes, nail

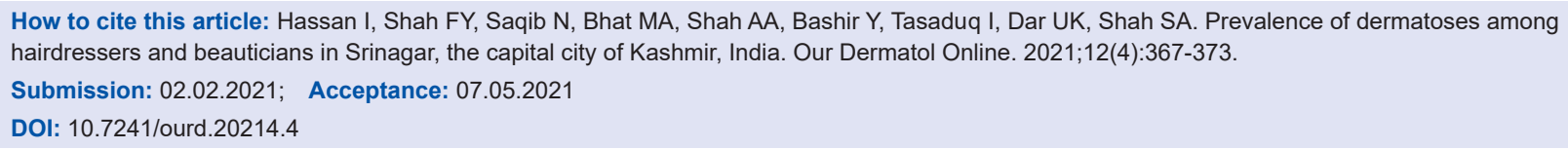


polishes, massage oils, and skin care products. Contact dermatitis is the dominant occupational dermatosis encountered and includes both irritant and allergic contact dermatitis [2]. Irritant dermatitis is attributed to wet work and exposure to irritants such as shampoos, conditioners, and styling products [3]. This results in delipidation of the skin leading to dry, flaked, split, and cracked skin. Ammonium persulfate in bleaches, para-phenylenediamine and para-aminoazobenzene in hair dyes, nickel sulfate and cobalt chloride in metals, balsam of Peru in fragrances, formaldehyde in preservatives, and glyceryl thioglycolate in wave components are the common allergens [4].

In India, hairdressers and beauticians work for long hours, take fewer precautions, and are less likely to be certified in their field of expertise when compared to those in Western countries. The licensing procedure is also less rigorous and the possibility of untrained professionals performing this sort of work is a valid concern. The distinction between hairdressers and beauticians is not clear-cut in India and both services are often provided by same person at a salon or parlor and, thus, the terms may be used interchangeably. The statistics regarding the prevalence of dermatoses among hairdressers and beauticians in the subcontinent are not plentiful. In this study, we attempted to determine the prevalence, clinical features, and pattern of dermatoses in hairdressers and beauticians in Srinagar, the capital city of Kashmir, India. We also aimed to educate and raise awareness among the study group regarding potential skin problems and relevant preventive measures.

\section{MATERIALS AND METHODS}

The study was an observational cross-sectional study conducted in the district Srinagar, the summer capital of Jammu and Kashmir. One hundred hairdressers and beauticians working in 57 salons and parlors located in Srinagar were included in the study. Institutional ethical committee clearance was acquired before recruiting the participants for the study. The sample size was decided after consultation with a statistician. The selection of salons for the study was performed randomly by multistage cluster sampling. The subjects were recruited during personal visits at randomly selected salons and parlors after explaining the purview of the study and obtaining a valid consent.

\section{Inclusion Criteria}

All hairdressers and beauticians working full-time for at least one year and willing to participate in the study were enrolled in the study.

\section{Exclusion Criteria}

Trainees and apprentices and those who did not consent to take part in the study were excluded.

The study participants were interviewed regarding their job description, work exposure, medical history, occupational history, and use of protective equipment such as gloves and aprons. Skin examination was performed in search of dermatoses. All the relevant data was recorded on predesigned proformas. Patch tests were performed with the Indian standard series on all participants, which consists of twenty antigens commonly implicated in the causation of allergic contact dermatitis (Table 1).

The antigens were placed on twenty patch test chambers mounted on two adhesive tapes. The adhesive tape along with the patch test chambers constituted a patch test unit. The two patch test units thus formed were stuck on the back of each participant (Fig. 1). The sites of application of the antigens were marked on the back with skin markers. The patch test units were removed after 48 hours and readings were taken at that time and again at 96 hours. The grading of the patch test results based on the morphology of the lesions as per the guidelines of The International Contact Dermatitis Research Group (ICDRG) (Table 2) [5].

\begin{tabular}{ll} 
Table 1: Antigens in the Indian standard series \\
\hline No. & Name of antigen \\
\hline 1 & Vaseline \\
2 & Wood alcohol (lanolin) \\
3 & Balsam of Peru \\
4 & Formaldehyde \\
5 & Mercaptobenzothiazole \\
6 & Potassium bichromate \\
7 & Nickel sulfate \\
8 & Cobalt sulfate \\
9 & Colophony \\
10 & Epoxy resins \\
11 & Paraben mix \\
12 & Paraphenylenediamine \\
13 & Parthenium \\
14 & Neomycin sulfate \\
15 & Benzocaine \\
16 & Chlorocresol \\
17 & Fragrance mix \\
18 & Thiuram mix \\
19 & Nitrofurazone \\
20 & Black rubber mix \\
\hline &
\end{tabular}


All the data was compiled in the form of a master chart and statistical analysis was done with the OpenEpi software. A p of less than 0.05 was defined as significant.

\section{RESULTS}

The study included a total of 100 participants - 54 males and 46 females. Most of the hairdressers and beauticians were nonlocals, comprising $59 \%$ of the study sample. The average age was 14 to 48 years, with a mean age of $24.3 \pm 6.3$ years (Table 3 ).

Out of the 100 participants, 54 (54\%) had one or more occupational dermatoses, while the remaining 46 were free of skin problems. The various dermatoses

Table 2: Grading of patch test results as per the guidelines of International Contact Dermatitis Research Group (ICDRG)

\begin{tabular}{ll}
\hline Morphological Symbol & Interpretation \\
\hline$-/ \quad$ No reaction & Negative \\
$? \quad$ Erythema only, no infiltration & Doubtful reaction \\
$+\quad$ Erythema, infiltration, and/or papules & Weakly positive \\
++ Erythema, infiltration, papules, and vesicles & Strongly positive \\
+++ Erythema, infiltration, and confluent vesicles & Extremely positive \\
IR Vesicles, blisters, and necrosis & Irritant reaction \\
NT & Not tested \\
\hline
\end{tabular}

Table 3: Demographic characteristics of our study sample

\begin{tabular}{lllcc}
\hline No. & Characteristic & Group & Number & Percentage \\
\hline 1 & Age & $10-20$ years old & 20 & $20 \%$ \\
& & $21-30$ years old & 68 & $68 \%$ \\
& & $31-40$ years old & 10 & $10 \%$ \\
& & $41-50$ years old & 2 & $2 \%$ \\
2 & Gender & Male & 54 & $54 \%$ \\
& & Female & 46 & $46 \%$ \\
3 & Professional & $<1$ year & 0 & $0 \%$ \\
& experience & $1-5$ years & 68 & $68 \%$ \\
& & $5-10$ years & 24 & $24 \%$ \\
& & $>10$ years & 8 & $8 \%$ \\
\hline
\end{tabular}

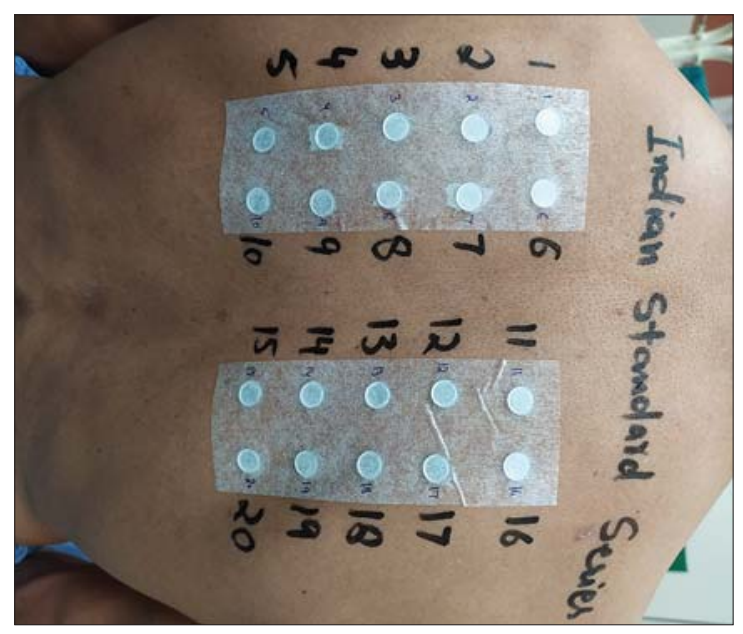

Figure 1: Patch test units applied on the back of a respondent. encountered during the course of the study are listed and described below.

\section{Hand Eczema}

Hand eczema was the most commonly encountered occupational dermatosis, observed in $41 \%$ of the cases. We found three common patterns of hand eczema: classic, interdigital, and threading. Classic eczema mostly involved the palmar and dorsal surface of the hands, while interdigital eczema involved the web spaces of the fingers, as the name suggests (Figs. 2 and 3). Threading eczema involved friction occurring as a result of a thread wound around the fingers during hair removal procedures (Fig. 4). Isolated classic hand eczema was seen in $20 \%$ of the cases, interdigital eczema in $9 \%$, and threading eczema in $8 \%$. A combination of classic with interdigital or threading eczema was seen each in $2 \%$ of the cases. Out of the 41 cases with hand eczema, only 13 cases $(31.7 \%$; $=13 / 41)$ used gloves

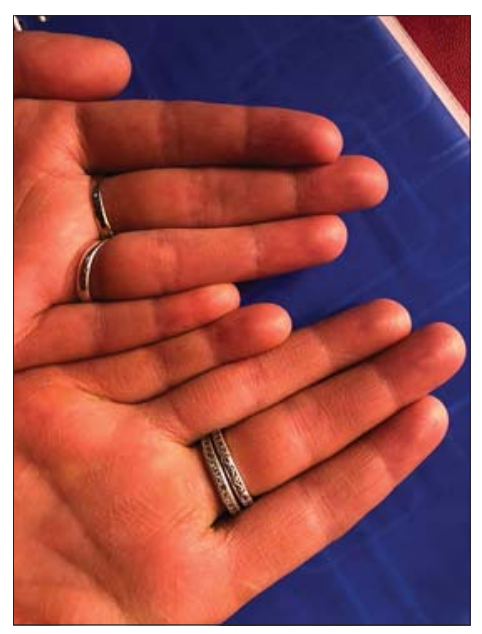

Figure 2: Classical hand eczema - Scaling and fissuring on the palms.

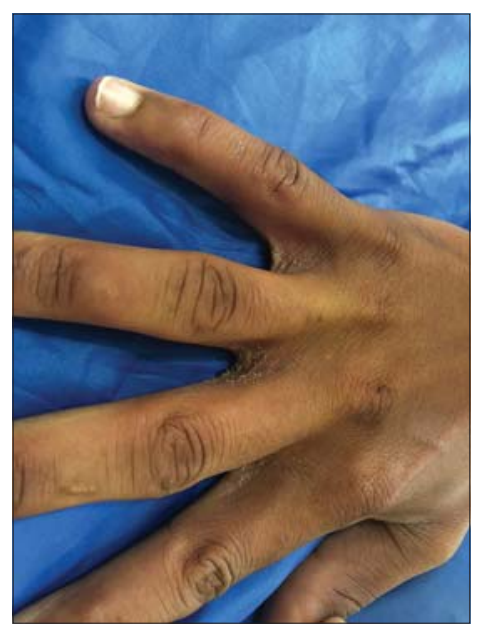

Figure 3: Interdigital eczema - Scaling involving the interdigital spaces predominantly. 
as a protective measure. However, none of the cases reported consistent and proper use of protective gloves. The use of gloves was, at best, sporadic and as needed. Among the 59 cases without hand eczema, 40 (67.8\%; n $=40 / 59$ ) reported proper and consistent use of gloves. The difference between the two groups was found to be statistically significant $(p=0.0003)$.

\section{Callosities}

Friction-induced callosities involving the hands were noted in 19 cases (19\%). The callosities were observed on the palmar surface of the hands in 2 cases (2\%) (Fig. 5). These might be attributed to the regular use of foot files for pedicure. However, the more common variety of callosities encountered was on the dorsal surface of the middle phalanx of the ring finger and, less commonly, on the thumb of the right hand, in 17 cases (17\%) (Fig. 6). We colloquially used the term

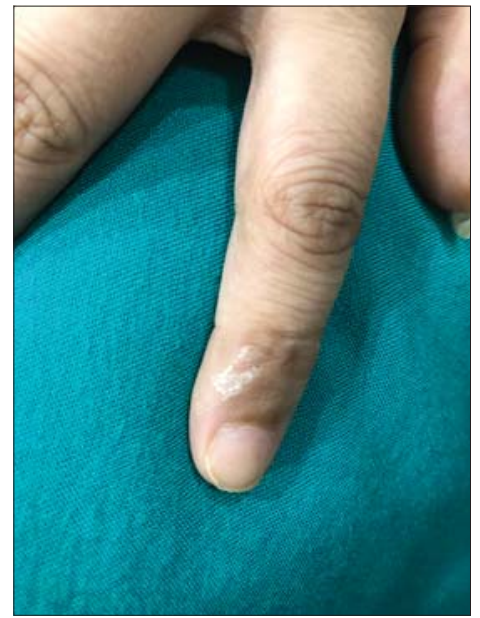

Figure 4: Threading eczema - Fissures with scaling on the lateral aspect of the index finger.

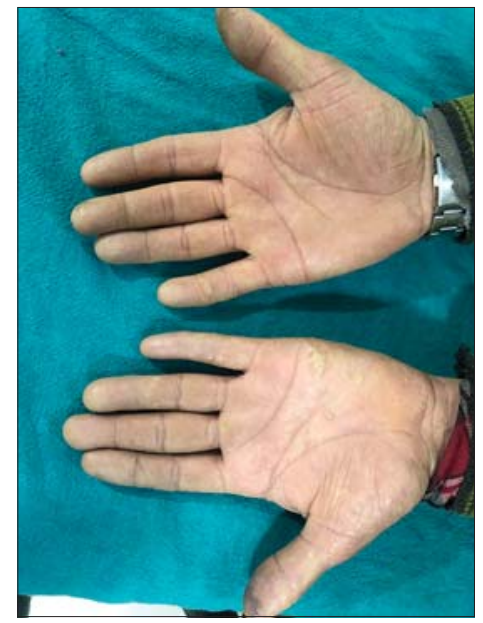

Figure 5: Callosities involving the palmar surface of hands. scissor nodules to refer to these lesions due to their typical location and nodular morphology.

\section{Nail Staining}

Staining of the distal portion of the nails was an occupation-related dermatosis encountered in 19 (19\%) participants involved in hair dying professionally, but only in cases in which the hairdresser or beautician preferred bare hands for the application of dyes (Fig. 7). The color of the nail stains varied from brownish-black to blue and could generally subside with time off work.

\section{PATCH TEST RESULTS}

Patch testing was performed in all cases, regardless of the presence or absence of a dermatosis reported. Out of the 100 cases, positive results were obtained in 35 (35\%), including 22 suffering from hand eczema and 13 without evidence of contact dermatitis. Out of the

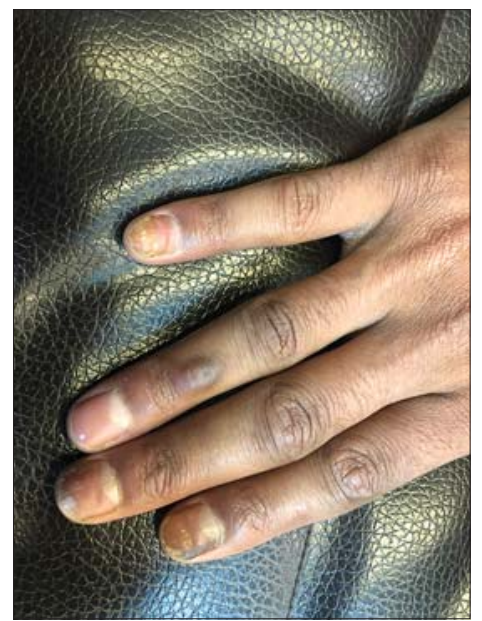

Figure 6: Scissor nodule - Nodular callosity on the dorsum of middle phalanx of ring finger.

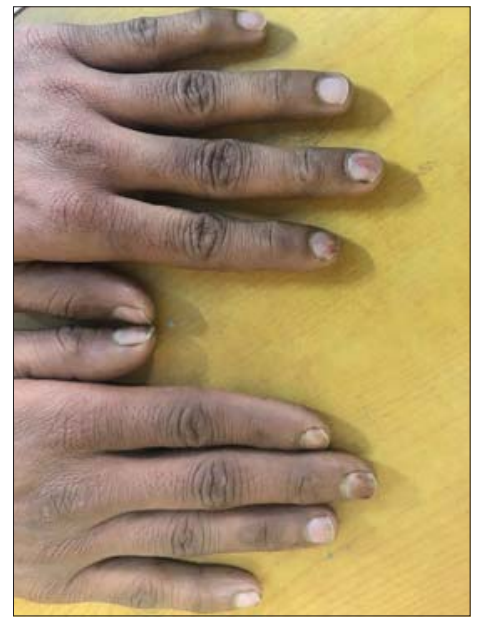

Figure 7: Nail staining - Bluish black staining of distal end of nails. 
41 cases with hand eczema, $22(53.7 \% ; n=22 / 41)$ gave a positive patch test, while, among the remaining 59 , only $13(22 \% ; n=13 / 59)$. The difference between the two groups was found to be statistically significant, pointing toward a strong possibility of allergic contact dermatitis in the former group $(p=0.001)$. A positive reaction in the group with hand eczema was observed in $16(80 \%$; $n=16 / 20)$ cases with classic eczema, $3(75 \% ; n=3 / 4)$ with combination eczema, 2 with interdigital eczema $(22.2 \% ; \mathrm{n}=2 / 9)$, 1 with threading eczema $(12.5 \% ; n=1 / 8)$. Patch testing was found to be the most relevant in the setting of classic hand eczema with the chi-squared test $(p=0.001)$. The most common allergens leading to a positive patch test were found to be paraphenylenediamine (PPD) in thirteen cases $(13 \% ; \mathrm{n}=13 / 100)$, followed by a fragrance mix in nine $(9 \% ; \mathrm{n}=9 / 100)$, nickel sulfate in nine $(9 \% ; \mathrm{n}=9 / 100)$, thiuram in three $(3 \% ; \mathrm{n}=3 / 100)$, and formaldehyde in one $(1 \% ; n=1 / 100)$. All the 22 cases who tested positive for PPD or a fragrance mix (Figs. 8 and 9) were suffering from hand eczema, while the 13 cases who tested positive for nickel sulfate or thiuram had no clinical evidence of eczema. Thus, a causal relationship between hypersensitivity and

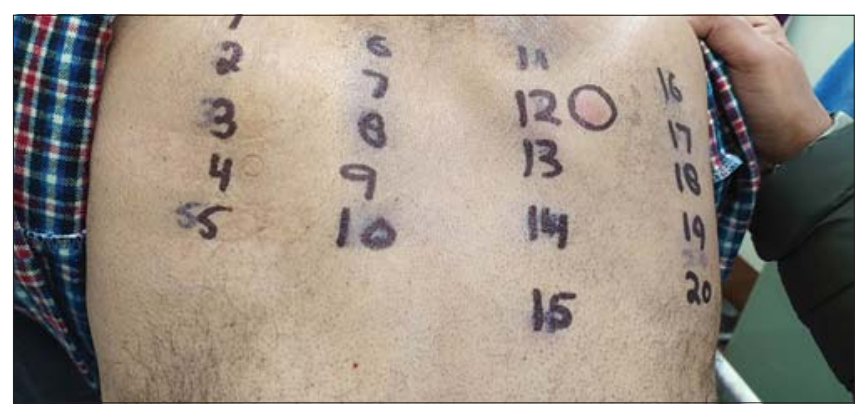

Figure 8: Patch test positive with erythema and induration at site 12 (Paraphenylene diamine).

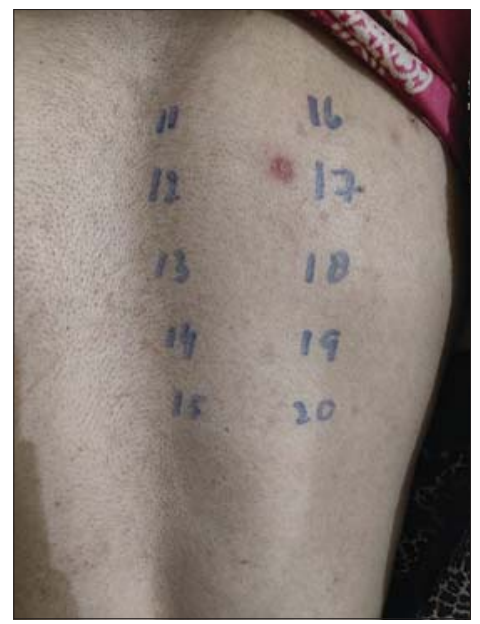

Figure 9: Patch test positive with erythema and induration at site 17 (Fragrance mix). eczema might be established in the case of PPD and a fragrance mix but, in the case of nickel sulfate, thiuram, and formaldehyde, there was hypersensitivity but no causal association with eczema.

\section{DISCUSSION}

Skin disorders are a common problem among hairdressers and beauticians, owing to the nature of their work and the regular exposure to chemicals. Out of the studied cases, $59 \%$ were found to suffer from one or more dermatoses. This was similar to a study from Dhaka, reporting the frequency of dermatoses among hairdressers to be $76.7 \%$, and to another study by Caroe et al., reporting the prevalence to be $46.7 \%[6,7]$.

Hand eczema was the most commonly encountered dermatosis and this was consistent with previous studies $[6,8,9]$. Three patterns of hand eczema were encountered: classic hand eczema, presenting with scaly plaques on the palmar surfaces of the hands, occurring due to exposure to a myriad of allergens; interdigital eczema, involving the web spaces of the fingers, seen in cases frequently involved in wet work such as shampooing clients and spa treatments, occurring typically due to the passage of hair between the hairdresser's fingers during these procedures, increasing exposure to irritants; and threading eczema, most commonly seen in the form of linear fissures with scaling and involving the lateral aspect of the distal phalanx of the index finger, occurring due to constant friction caused by a thread twisted around the index finger in threading procedures such as hair removal. The role of mechanical friction in the development and aggravation of eczema is a well-known fact, documented in a number of studies $[10,11]$. We also witnessed a similar pattern. Based on history taking, work patterns, and the clinical presentation, it may likely be presumed that interdigital eczema is irritant in nature, classic hand eczema is allergic in nature, while threading eczema is frictional in nature.

Proper and consistent use of gloves during all procedures offered good protection against the development of eczema, as documented by a statistically significant difference observed in our study. Although sensitivity to thiuram was documented by patch testing in some cases, no clinical significance could be assigned to this finding.

Callosities were commonly encountered among the study subjects and occurred due to constant friction. 
Callosities involving the palmar surfaces of the hands were seen in a minority of the cases (Fig. 5), while the more commonly encountered pattern involved the dorsum of the distal phalanx of the ring finger and thumb, colloquially termed scissor nodules (Fig. 6). These occurred on the dominant hand due to hairdressers' typical habit of holding the scissors by the thumb and ring finger and occurred classically in cases in the habit of using scissors with iron finger rings. Scissors with plastic finger rings were less likely to cause scissor nodules.

Staining of the distal portion of the nails was another problem encountered among the hairdressers and beauticians (Fig. 7). It was associated with the use of coloring agents and was found exclusively in cases lacking consistency in the use of gloves during hair dying. Most were uncomfortable with the use of gloves and believed that applying dyes directly with bare hands resulted in a better final result and more customer satisfaction. The staining was of a semipermanent nature as it was resistant to washing, even repeatedly, but would improve over a couple of months off work.

Patch testing was done in all cases, regardless of the presence or absence of eczema. Among the studied participants, 35\% (n=35/100) tested positive. This was comparable with the findings reported by a Polish and a Danish study, reporting positive patch test results in $38.1 \%$ and $55.1 \%$ of cases, respectively. $[12,13]$ Although Gupta et al. reported a much higher positivity of patch test results in their study $(73.3 \%)$, this might be attributed to the fact that they did patch testing only in cases with suspected contact dermatitis [14]. Classic hand eczema showed a statistically significant relation with patch test positivity. Out of the three patterns of hand eczema noted in our study, classic hand eczema was the most likely to be allergic in nature. This was consistent with the clinical assessment that interdigital eczema is related to excessive wet work with shampoos and chemicals while threading eczema is related to friction. Out of all 41 cases of eczema, $53.7 \%(\mathrm{n}=22 / 41)$ were confirmed as allergic in nature, based on history taking, clinical examination, and patch testing, while $21.9 \%(n=9 / 41)$ were cases of irritant dermatitis. This finding was in consonance with a previous study by Lyons et al. [15], who reported the prevalence of allergic and irritant contact dermatitis to be $71 \%$ and $20 \%$, respectively.

The most common allergens of occupational relevance determined by patch testing were paraphenylenediamine (13\%), followed by a fragrance mix (9\%), and thiuram (3\%). Although thiuram sensitization might be attributed to the use of gloves, no clinical relevance could be attributed to this finding. Antigens such as nickel sulfate (9\%) and formaldehyde $(1 \%)$ also showed a positive reaction in certain cases but these were unlikely to bear clinical relevance. Our findings were similar to those reported by Gupta and Minamoto, who found the most common allergens to be paraphenylenediamine, followed by a fragrance mix $[15,16]$. A study by Krecisz et al. documented nickel sulfate to be the most common sensitizer (29.3\%) [12]. While it was much less so, their study included more than $90 \%$ of females, while ours had a more balanced distribution, with approx. $50 \%$ of females.

Skin disorders are a prevalent health problem among hairdressers and beauticians in the region of India, affecting more than $50 \%$ of respondents. Proper and consistent use of protective equipment such as gloves and the avoidance of prolonged exposure to irritants and allergens should be promoted in this group. With proper education and institution of protective measures, the prevalence of dermatoses in this vulnerable group may be reduced greatly.

\section{Limitations}

We used no cosmetic series when patch testing our study sample, which could have been more informative, and the sample size in our study was small.

\section{CONCLUSION}

The incidence of cosmetic dermatitis is high among beauticians and hairdressers. Hair dyes, creams, and shampoos are the commonly implicated agents in the causation of eczema, whereas paraphenylenediamine and a fragrance mix are the most common causative allergens. Callosities and nail staining are also encountered commonly, although these are not especially bothersome to the patient. Recommendation regarding the proper precautions and protective equipment for this group of professionals is needed.

\section{Statement of Human and Animal Rights}

All the procedures followed were in accordance with the ethical standards of the responsible committee on human experimentation (institutional and national) and with the 2008 revision of the Declaration of Helsinki of 1975. 


\section{Statement of Informed Consent}

Informed consent for participation in this study was obtained from all patients.

\section{REFERENCES}

1. Seck B, Ndiaye MT, Diop A, Gaye C, Diouf A, Diagne FG, et al. The relevancy of patch testing in the exploration of the cutaneous side effects of herbal medicine. Our Dermatol Online. 2021;12:19-23.

2. Lyons G, Roberts H, Palmer A, Matheson M, Nixon R. Hairdressers presenting to an occupational dermatology clinic in Melbourne, Australia. Contact Dermatitis. 2013;68:300-6.

3. Faghihi G, Radan Y, Radan MR. Irritant hand dermatitis during the COVID-19 outbreak. Our Dermatol Online. 2020;11(Supp. 2):15-6.

4. Wang MZ, Farmer SA, Richardson DM, Davis MD. Patch-testing with hairdressing chemicals. Dermatitis. 2011;22:16-26.

5. Parajuli S, Paudel V, Paudel U, Pokhrel DB. Pattern of patch test reactivity among patients with clinical diagnosis of contact dermatitis: A hospital-based study. Our Dermatol Online. 2017;8:389-92.

6. Islam MDS, Ahmed UM, Firoz AMD, Ullah MF, Mortaz RE, Faruquee MH. Occupational dermatitis among the hair dressers of selected area of Dhaka city. MOJ Public Health. 2015;2:61-3.

7. Carøe TK, Ebbehøj NE, Agner T. Occupational dermatitis in hairdressers - Influence of individual and environmental factors. Contact Dermatitis. 2017; 76: 146-50.

8. Gupta M. Hand eczema and patch testing - A clinico-allergiological study. Our Dermatol Online. 2019;10:255-8.

9. Zheleva D, Darlenski R. Occupational fingertip eczema from acrylates in a manicurist. Our Dermatol Online. 2015;6:204-6.

10. Lawton S. Understanding hand eczema: Features, diagnosis and treatment. Independent Nurse. 2018;2018:28-30.

11. Politiek K, Loman L, Pas HH, Diercks GFH, Lemmink HH, Jan SZ, et al. Hyperkeratotic hand eczema: Eczema or not? Contact Dermatitis. 2020;83:196-205.

12. Krecisz B, Kiec-Swierczynska M, Chomiczewska D. Dermatological screening and results of patch testing among Polish apprentice hairdressers. Contact Dermatitis. 2011;64:90-5.

13. Schwensen JF, Johansen JD, Veien NK, Funding AT, Avnstorp C, Osterballe $\mathrm{M}$, et al. Occupational contact dermatitis in hairdressers: An analysis of patch test data from the Danish contact dermatitis group, 2002-2011. Contact Dermatitis. 2014;70:233-7.

14. Gupta M. Cosmetic contact sensitivity among beauticians and hairdressers: A clinicoepidemiological study. Egypt J Dermatol Venerol. 2017;37:7-10.

15. Lyons G, Roberts H, Palmer A, Matheson M, Nixon R. Hairdressers presenting to an occupational dermatology clinic in Melbourne, Australia. Contact Dermatitis. 2013;68:300-6.

16. Minamoto K. [Skin sensitizers in cosmetics and skin care products]. Nihon Eiseigaku Zasshi. 2010;65:20-9.

Copyright by Iffat Hassan, et al. This is an open access article distributed under the terms of the Creative Commons Attribution License, which permits unrestricted use, distribution, and reproduction in any medium, provided the original author and source are credited.

Source of Support: Nil, Conflict of Interest: None declared. 